\title{
A Study on the Chain Ratio-Type Estimator of Finite Population Variance
}

\author{
Yunusa Olufadi ${ }^{1}$ and Cem Kadilar ${ }^{2}$ \\ ${ }^{1}$ Department of Statistics and Mathematical Sciences, Kwara State University, PMB 1530, Malete, Ilorin, Nigeria \\ ${ }^{2}$ Department of Statistics, Hacettepe University, Beytepe, 06800 Ankara, Turkey
}

Correspondence should be addressed to Yunusa Olufadi; olufadil4yunus@yahoo.com

Received 12 August 2013; Revised 15 January 2014; Accepted 15 January 2014; Published 24 February 2014

Academic Editor: Shein-chung Chow

Copyright (C) 2014 Y. Olufadi and C. Kadilar. This is an open access article distributed under the Creative Commons Attribution License, which permits unrestricted use, distribution, and reproduction in any medium, provided the original work is properly cited.

We suggest an estimator using two auxiliary variables for the estimation of the unknown population variance. The bias and the mean square error of the proposed estimator are obtained to the first order of approximations. In addition, the problem is extended to two-phase sampling scheme. After theoretical comparisons, as an illustration, a numerical comparison is carried out to examine the performance of the suggested estimator with several estimators.

\section{Introduction}

Variations are present everywhere in our daily life. It is the law of nature that no two things or individuals are exactly alike. For instance, a physician needs a full understanding of variations in the degree of human blood pressure, body temperature, and pulse rate for adequate prescription. A manufacturer needs constant knowledge of the level of variations in people's reaction to his product to be able to know whether to reduce or increase his price or improve the quality of his product. An agriculturist needs an adequate understanding of the variations in climatic factors especially from place to place (or time to time) to be able to plan on when, how, and where to plant his crop.

It is well known that the use of auxiliary information in sample survey designs results in efficient estimators of population parameters, such as variance, under some realistic conditions. For example, when information is available on the auxiliary variable that is positively correlated with the study variable, the ratio estimator is a suitable estimator for the estimation of the population variance.

Let $P$ be a finite population consisting of $N$ units, $P_{1}, P_{2}$, $\ldots, P_{N}$. The units of this finite population are identifiable in the sense that they are uniquely labeled from 1 to $N$ and the label on each unit is known. Let $y$ be the character under study taking the value $y_{i}$ on the units $P_{i}(i=1,2, \ldots, N)$ and assume a sample of size $n$ is drawn by the simple random sampling without replacement (SRSWOR).

Suppose in a survey problem that we are interested in estimating the population variance, $S_{y}^{2}$. Isaki [1] presented the ratio estimator for the population variance using the auxiliary information. The problem of estimating the population variance using information on single auxiliary variable has also been discussed by various authors including Prasad and Singh [2, 3], Biradar and Singh [4], Rueda Garcia and Arcos Cebrian [5], Arcos et al. [6], Kadilar and Cingi [7], and Singh et al. [8].

The mean square error (MSE) of the classical estimator of the population variance, $S_{y}^{2}$, which we denote as $t_{0}$, is $V\left(t_{0}\right)=$ $S_{y}^{4} A_{0}$. Quite often, information on many auxiliary variables is available in the survey which can be utilized to increase the precision of the estimate. The ratio estimator of population variance for a single auxiliary variable denoted as $t_{1}$ suggested by Isaki [1] and the two-phase sampling (TPS) estimator of $t_{1}$ denoted as $t_{1}^{*}$ are as follows:

$$
\begin{gathered}
t_{1}=s_{y}^{2} \frac{S_{x 1}^{2}}{s_{x 1}^{2}}, \\
\operatorname{MSE}\left(t_{1}\right)=S_{y}^{4}\left(A_{0}+A_{1}-2 A_{3}\right),
\end{gathered}
$$




$$
\begin{gathered}
t_{1}^{*}=s_{y}^{2} \frac{s_{x 1}^{* 2}}{s_{x 1}^{2}}, \\
\operatorname{MSE}\left(t_{1}^{*}\right)=\operatorname{MSE}\left(t_{1}\right)-S_{y}^{4}\left(A_{1}^{*}-2 A_{3}^{*}\right) .
\end{gathered}
$$

Following Olkin [9], Isaki [1] also presented the ratio estimator of variance using two auxiliary variables as follows:

$$
\begin{gathered}
t_{2}=W_{1} \frac{s_{y}^{2}}{s_{x 1}^{2}} S_{x 1}^{2}+W_{2} \frac{s_{y}^{2}}{s_{x 1}^{2}} S_{x 2}^{2}, \\
\operatorname{MSE}\left(t_{2}\right)=S_{y}^{4}\left(C_{1}+W_{1}^{2} C_{2}-2 W_{1} C_{3}\right), \\
t_{2}^{*}=M_{1} \frac{s_{y}^{2}}{s_{x 1}^{2}} s_{x 1}^{* 2}+M_{2} \frac{s_{y}^{2}}{s_{x 2}^{2}} s_{x 2}^{* 2} \quad\left(\text { TPS approach of } t_{2}\right), \\
\operatorname{MSE}\left(t_{2}^{*}\right)=S_{y}^{4}\left(D_{1}+M_{1}^{2} D_{2}-2 M_{1} D_{3}\right),
\end{gathered}
$$

where $W_{i}$ and $M_{i}$, for $i=1,2$, are weights chosen to minimize the MSE of $t_{2}$ and $t_{2}^{*}$. Further, $\sum W_{i}=1$ and $\sum M_{i}=1$, where

$$
\begin{aligned}
& s_{x 1}^{2}=\frac{1}{n} \sum_{i=1}^{n}\left(x_{1 i}-\bar{X}_{1}\right)^{2}, \quad s_{x 2}^{* 2}=\frac{1}{n} \sum_{i=1}^{n}\left(x_{2 i}-\bar{X}_{2}\right)^{2}, \\
& s_{x 1}^{* 2}=\frac{1}{n^{\prime}} \sum_{i=1}^{n^{\prime}}\left(x_{1 i}-\bar{x}_{1}^{\prime}\right)^{2}, \quad s_{x 2}^{* 2}=\frac{1}{n^{\prime}} \sum_{i=1}^{n^{\prime}}\left(x_{2 i}-\bar{x}_{2}^{\prime}\right)^{2} \text {, } \\
& \bar{x}_{1}^{\prime}=\frac{1}{n^{\prime}} \sum_{i=1}^{n^{\prime}} x_{1 i}, \quad \bar{x}_{2}^{\prime}=\frac{1}{n^{\prime}} \sum_{i=1}^{n^{\prime}} x_{2 i}, \\
& C_{1}=A_{0}+A_{2}-2 A_{4}, \quad C_{1}^{*}=A_{2}^{*}-2 A_{4}^{*}, \\
& D_{1}=C_{1}-C_{1}^{*}, \quad C_{2}=A_{1}+A_{2}-2 A_{5}, \\
& C_{2}^{*}=A_{1}^{*}+A_{2}^{*}-2 A_{5}^{*}, \quad D_{2}=C_{2}-C_{2}^{*} \text {, } \\
& C_{3}=A_{2}+A_{3}-A_{4}-A_{5}, \quad C_{3}^{*}=A_{2}^{*}+A_{3}^{*}-A_{4}^{*}-A_{5}^{*} \text {, } \\
& D_{3}=C_{3}-C_{3}^{*} \\
& A_{0}=\frac{1}{n}\left(\lambda_{400}-1\right), \quad A_{1}=\frac{1}{n}\left(\lambda_{040}-1\right), \\
& A_{2}=\frac{1}{n}\left(\lambda_{004}-1\right), \quad A_{3}=\frac{1}{n}\left(\lambda_{220}-1\right), \\
& A_{4}=\frac{1}{n}\left(\lambda_{202}-1\right), \quad A_{5}=\frac{1}{n}\left(\lambda_{022}-1\right), \\
& A_{1}^{*}=\frac{1}{n^{\prime}}\left(\lambda_{040}-1\right), \quad A_{2}^{*}=\frac{1}{n^{\prime}}\left(\lambda_{004}-1\right), \\
& A_{3}^{*}=\frac{1}{n^{\prime}}\left(\lambda_{220}-1\right), \quad A_{4}^{*}=\frac{1}{n^{\prime}}\left(\lambda_{202}-1\right), \\
& A_{5}^{*}=\frac{1}{n^{\prime}}\left(\lambda_{022}-1\right) \text {, }
\end{aligned}
$$

$$
\begin{gathered}
\lambda=\frac{\mu_{a b c}}{\mu_{200}^{a / 2} \mu_{020}^{b / 2} \mu_{002}^{c / 2}} \\
\mu_{a b c}=\frac{1}{N-1} \sum_{i=1}^{N}\left(y_{i}-\bar{Y}\right)^{a}\left(x_{1 i}-\bar{X}_{1}\right)^{b}\left(x_{2 i}-\bar{X}_{2}\right)^{c}
\end{gathered}
$$

where $a, b$, and $c$ are nonnegative integers.

Several authors (Srivastava et al. [10], Upadhyaya et al. [11], and Singh et al. [12]) adopted TPS procedure proposed by Chand [13] and have suggested some chain ratio-type estimators for estimating population mean $\bar{Y}$ of $y$. In the same vein, Gupta et al. [14] and Singh et al. [8] proposed the following classes of estimators under the assumption that the population variance of the first auxiliary variable $S_{x 1}^{2}$ is not known, but the population variance of another auxiliary variable $X_{2}$ closely related to $X_{1}$ is available. The MSEs of the estimators suggested by Gupta et al. [14] and Singh et al. [8] are, respectively, given by

$$
\begin{gathered}
t_{3}=s_{y}^{2}\left(\frac{s_{x 1}^{2}}{s_{x 1}^{* 2}}\right)^{I_{1}}\left(\frac{s_{x 2}^{* 2}}{s_{x 2}^{2}}\right)^{I_{2}}, \\
\mathrm{MSE}_{\text {min }}\left(t_{3}\right)=s_{y}^{4}\left[A_{0}-\phi\left(\frac{A_{3}^{2}}{A_{1}}\right)-\left(\frac{A_{4}^{2}}{n^{\prime} A_{2}}\right)\right], \\
t_{4}=s_{y}^{2}\left(\frac{s_{x 1}^{2}}{s_{x 1}^{* 2}}\right)^{J_{1}}\left(\frac{s_{x 2}^{* 2}}{s_{x 2}^{2}}\right)^{J_{2}}\left(\frac{s_{x 2}^{2}}{S_{x 2}^{2}}\right)^{J_{3}}, \\
\operatorname{MSE}_{\text {min }}\left(t_{4}\right)=s_{y}^{4} A_{0}\left[1-\delta \gamma_{0.12}^{* 2}-\theta \rho^{* 2}\right],
\end{gathered}
$$

where $I_{1}, I_{2}$, and $J_{i}$ for $i=1,2,3$ are constants chosen to minimize the MSE of $t_{3}$ and $t_{4} ; \phi=\left(1 / n-1 / n^{\prime}\right) ; \theta=n / n^{\prime} ; \delta=$ $\left(\left(n^{\prime}-n\right) / n^{\prime}\right) ; \gamma_{0.12}^{* 2}=\left(A_{2} A_{3}^{2}-2 A_{3} A_{4} A_{5}+A_{1} A_{4}^{2}\right) / A_{0}\left(A_{1} A_{2}-\right.$ $\left.A_{5}^{2}\right) ; \rho^{* 2}=A_{4}^{2} / A_{0} A_{2}$.

In most studies, several variables are considered simultaneously either to explain or estimate (predict) the study variable. In most cases, information on several auxiliary variables closely related to the study variable may be easily obtained on all units in the population. For example, while conducting an educational survey, the investigator may be interested in studying characteristics such as age, gender, hours spent on studying per day, sitting position, parent's educational level, parent's income, relationship with lectures and access to facilities (e.g., library, internet, laboratory), among others. With the main aim of suggesting a more efficient estimator, we propose in this paper, under SRSWOR, a chain ratio-type estimator for estimating the population variance when information on two auxiliary variables is available. In addition, the problem is extended to the case of TPS.

\section{The Suggested Estimator}

Following Abu-Dayyeh et al. [15], we define an estimator for estimating the population variance, $S_{y}^{2}$, as follows:

$$
t=s_{y}^{2}\left(\frac{S_{x 1}^{2}}{s_{x 1}^{2}}\right)^{\alpha_{1}}\left(\frac{S_{x 2}^{2}}{s_{x 2}^{2}}\right)^{\alpha_{2}},
$$

where $\alpha_{1}$ and $\alpha_{2}$ are real constants to be determined such that the MSE of $t$ is minimum. 
To determine the bias and MSE of $t$, we define

$$
s_{y}^{2}=S_{y}^{2}\left(1+k_{0}\right) ; \quad s_{x 1}^{2}=S_{x 1}^{2}\left(1+k_{1}\right) ; \quad s_{x 2}^{2}=S_{x 2}^{2}\left(1+k_{2}\right),
$$

such that

$$
\begin{gathered}
E\left(k_{0}\right)=E\left(k_{1}\right)=E\left(k_{2}\right)=0, \\
E\left(k_{0}^{2}\right)=A_{0}, \quad E\left(k_{1}^{2}\right)=A_{1}, \\
E\left(k_{2}^{2}\right)=A_{2}, \quad E\left(k_{0} k_{1}\right)=A_{3}, \\
E\left(k_{0} k_{2}\right)=A_{4}, \quad E\left(k_{1} k_{2}\right)=A_{5},
\end{gathered}
$$

Now, expressing $t$ in terms of $k$ 's, we have

$$
\begin{aligned}
t= & S_{y}^{2}\left(1+k_{0}\right)\left(1+k_{1}\right)^{-\alpha_{1}}\left(1+k_{2}\right)^{-\alpha_{2}} \\
= & S_{y}^{2}\left(1+k_{0}\right)\left(1-\alpha_{1} k_{1}+\frac{\alpha_{1}\left(\alpha_{1}+1\right)}{2} k_{1}^{2}\right) \\
& \times\left(1-\alpha_{2} k_{2}+\frac{\alpha_{2}\left(\alpha_{2}+1\right)}{2} k_{2}^{2}\right) .
\end{aligned}
$$

We assume that $\left|k_{1}\right|<1$ and $\left|k_{2}\right|<1$ so that $\left(1+k_{1}\right)^{-1}$ and $\left(1+k_{2}\right)^{-1}$ are expandable in terms of $k$ 's. By expanding the right hand side of (8), multiplying, and neglecting terms involving power of $k$ 's greater than two, we have

$$
\begin{aligned}
t-S_{y}^{2}=S_{y}^{2}( & k_{0}-\alpha_{1} k_{1}-\alpha_{2} k_{2}+\alpha_{1} \alpha_{2} k_{1} k_{2}-\alpha_{1} k_{0} k_{1} \\
& \left.-\alpha_{2} k_{0} k_{2}+\frac{\alpha_{1}\left(\alpha_{1}+1\right)}{2} k_{1}^{2}+\frac{\alpha_{2}\left(\alpha_{2}+1\right)}{2} k_{2}^{2}\right) .
\end{aligned}
$$

Taking expectations on both sides of (9), we get the bias of $t$, to the first degree of approximation, as

$$
B(t)=S_{y}^{2}\left(\frac{\alpha_{1}^{2}}{2} A_{1}+\frac{\alpha_{2}^{2}}{2} A_{2}+\alpha_{1} \alpha_{2} A_{5}-\alpha_{1} A_{3}-\alpha_{2} A_{4}\right) .
$$

Squaring both sides of (9) and neglecting terms of $k$ 's involving power greater than two, we have

$$
\begin{array}{r}
\left(t-S_{y}^{2}\right)^{2}=S_{y}^{4}\left(k_{0}^{2}+2 \alpha_{1} \alpha_{2} k_{1} k_{2}-2 \alpha_{1} k_{0} k_{1}\right. \\
\left.-2 \alpha_{2} k_{0} k_{2}+\alpha_{1}^{2} k_{1}^{2}+\alpha_{2}^{2} k_{2}^{2}\right) .
\end{array}
$$

Taking expectations on both sides of (11), we get the MSE of $t$, to the first order of approximation, as

$$
\begin{gathered}
\operatorname{MSE}(t)=S_{y}^{4}\left(A_{0}+\alpha_{1}^{2} A_{1}+\alpha_{2}^{2} A_{2}-2 \alpha_{1} A_{3}\right. \\
\left.-2 \alpha_{2} A_{4}+2 \alpha_{1} \alpha_{2} A_{5}\right) .
\end{gathered}
$$

The optimal values of $\alpha_{1}$ and $\alpha_{2}$ in (12) could be obtained by differentiating (12) with respect to $\alpha_{1}$ and $\alpha_{2}$ and equalizing to zero. After a little algebraic simplification, we have

$$
\alpha_{1}^{*}=\frac{A_{2} A_{3}-A_{4} A_{5}}{A_{1} A_{2}-A_{5}^{2}}, \quad \alpha_{2}^{*}=\frac{A_{1} A_{4}-A_{3} A_{5}}{A_{1} A_{2}-A_{5}^{2}} .
$$

We can obtain the minimum MSE of $t$ by simply substituting the optimal equations of $\alpha_{1}$ and $\alpha_{2}$ in (12).

\section{Suggested Estimator in TPS}

In certain practical situations, when $S_{x}^{2}$ is not also known, the technique of TPS sometimes referred to as double sampling is used. This scheme requires the collection of information on $x_{1}$ and $x_{2}$ in the first phase sample $s^{\prime}$ of size $n^{\prime}\left(n^{\prime}<N\right)$ and on $y$ for the second phase sample $s$ of size $n\left(n<n^{\prime}\right)$. The estimator $t^{*}$ in TPS will take the following form:

$$
t^{*}=s_{y}^{2}\left(\frac{s_{x 1}^{* 2}}{s_{x 1}^{2}}\right)^{\alpha_{3}}\left(\frac{s_{x 2}^{* 2}}{s_{x 2}^{2}}\right)^{\alpha_{4}} .
$$

To obtain the bias and MSE of $t^{*}$, we write

$$
\begin{gathered}
s_{y}^{2}=S_{y}^{2}\left(1+k_{0}\right), \quad s_{x 1}^{2}=S_{x 1}^{2}\left(1+k_{1}\right), \\
s_{x 1}^{* 2}=S_{x 1}^{2}\left(1+k_{1}^{*}\right), \quad s_{x 2}^{2}=S_{x 2}^{2}\left(1+k_{2}\right), \\
s_{x 2}^{* 2}=S_{x 2}^{2}\left(1+k_{2}^{*}\right),
\end{gathered}
$$

Note that

$$
\begin{gathered}
E\left(k_{1}^{*}\right)=E\left(k_{2}^{*}\right)=0, \quad E\left(k_{1}^{* 2}\right)=A_{1}^{*}, \\
E\left(k_{2}^{* 2}\right)=A_{2}^{*}, \quad E\left(k_{1} k_{1}^{*}\right)=A_{1}^{*}, \\
E\left(k_{2} k_{2}^{*}\right)=A_{2}^{*}, \quad E\left(k_{0} k_{1}^{*}\right)=A_{3}^{*}, \\
E\left(k_{0} k_{2}^{*}\right)=\frac{1}{n^{\prime}}\left(\lambda_{202}-1\right)=A_{4}^{*}, \\
E\left(k_{1} k_{1}^{*}\right)=E\left(k_{2} k_{1}^{*}\right)=E\left(k_{1}^{*} k_{2}^{*}\right)=A_{5}^{*} .
\end{gathered}
$$

Expressing $t^{*}$ in terms of $k$ 's and following the procedure explained in Section 2, we get the bias and MSE of the estimator, $t^{*}$, respectively, as

$$
\begin{gathered}
B\left(t^{*}\right)=S_{y}^{2}\left(\frac{\alpha_{3}\left(\alpha_{3}+1\right)}{2} E_{3}+\frac{\alpha_{4}\left(\alpha_{4}+1\right)}{2} E_{4}\right. \\
\left.+\alpha_{3} \alpha_{4} E_{5}-\alpha_{3} E_{1}-\alpha_{4} E_{2}\right), \\
\operatorname{MSE}\left(t^{*}\right)=\operatorname{MSE}(t)-S_{y}^{4}\left(\alpha_{3}^{2} A_{1}^{*}+\alpha_{4}^{2} A_{2}^{*}+2 \alpha_{3} \alpha_{4} A_{5}^{*}\right. \\
\left.+2 \alpha_{3} A_{3}^{*}+2 \alpha_{4} A_{4}^{*}\right),
\end{gathered}
$$

where

$$
\begin{gathered}
E_{1}=A_{3}-A_{3}^{*}, \quad E_{2}=A_{4}-A_{4}^{*}, \\
E_{3}=A_{1}-A_{1}^{*}, \quad E_{4}=A_{2}-A_{2}^{*}, \\
E_{5}=A_{5}-A_{5}^{*} .
\end{gathered}
$$


Minimization of (17), with respect to $\alpha_{3}$ and $\alpha_{4}$, yields their optimum values as

$$
\alpha_{3}^{*}=\frac{E_{1} E_{4}-E_{2} E_{5}}{E_{3} E_{4}-E_{5}^{2}}, \quad \alpha_{4}^{*}=\frac{E_{2} E_{3}-E_{1} E_{5}}{E_{3} E_{4}-E_{5}^{2}} .
$$

Substitution of $\alpha_{3}^{*}$ and $\alpha_{4}^{*}$ in (17) gives the minimum value of the MSE of $t^{*}$.

\section{Efficiency Comparisons}

In this section, we considered the theoretical comparisons of the performances of the suggested estimators $\left(t\right.$ and $\left.t^{*}\right)$ with respect to the traditional estimator $\left(t_{0}\right)$, Isaki [1] ratio estimators $t_{1}, t_{1}^{*}, t_{2}$, and $t_{2}^{*}$ (for single and double auxiliary variables), Gupta et al. [14] estimator, $\left(t_{3}\right)$ and Singh et al. [8] estimator $\left(t_{4}\right)$ which are investigated. We have the following conditions:

(i) $\operatorname{MSE}(t)-\operatorname{MSE}\left(t_{0}\right)<0 \Longrightarrow H_{1}<0$,

(ii) $\operatorname{MSE}(t)-\operatorname{MSE}\left(t_{1}\right)<0 \Longrightarrow H_{2}<0$,

(iii) $\operatorname{MSE}(t)-\operatorname{MSE}\left(t_{2}\right)<0 \Longrightarrow H_{3}+H_{4}<0$,

(iv) $\operatorname{MSE}(t)-\operatorname{MSE}\left(t_{3}\right)<0 \Longrightarrow H_{5}<H_{6}$,

(v) $\operatorname{MSE}(t)-\operatorname{MSE}\left(t_{4}\right)<0 \Longrightarrow H_{5}<H_{7}$,

(vi) $\operatorname{MSE}\left(t^{*}\right)-\operatorname{MSE}\left(t_{1}^{*}\right)<0$

$$
\Longrightarrow \operatorname{MSE}(t)-\operatorname{MSE}\left(t_{1}\right)<S_{y}^{4} H_{8} \text {, }
$$

(vii) $\operatorname{MSE}\left(t^{*}\right)-\operatorname{MSE}\left(t_{2}^{*}\right)<0$

$$
\Longrightarrow \operatorname{MSE}(t)-\operatorname{MSE}\left(t_{2}\right)<S_{y}^{4} H_{9} \text {, }
$$

where

$$
\begin{aligned}
H_{1}= & \alpha_{1}^{2} A_{1}+\alpha_{2}^{2} A_{2}+2 \alpha_{1} \alpha_{2} A_{5}-2 \alpha_{1} A_{3}-2 \alpha_{2} A_{4}, \\
H_{2}= & \left(\alpha_{1}^{2}-1\right) A_{1}+\alpha_{2}^{2} A_{2}-2\left(\alpha_{1}-1\right) A_{3} \\
& -2 \alpha_{2} A_{4}+2 \alpha_{1} \alpha_{2} A_{5}, \\
H_{3}= & \left(\alpha_{1}-W_{1}\right)\left[\left(\alpha_{1}+W_{1}\right) A_{1}-2 A_{3}\right] \\
& +\left(\alpha_{2}+W_{1}\right)\left[\left(\alpha_{2}-W_{1}\right) A_{2}-2 A_{4}\right]+2 A_{4}, \\
H_{4}= & 2\left(\alpha_{1} \alpha_{2}+W_{1}^{2}-W_{1}\right) A_{5}-\left(1-2 W_{1}\right) A_{2}, \\
H_{5}= & \alpha_{1}^{* 2} A_{1}+\alpha_{2}^{* 2} A_{2}-2 \alpha_{1}^{*} A_{3}-2 \alpha_{2}^{*} A_{4}+2 \alpha_{1}^{*} \alpha_{2}^{*} A_{5}, \\
H_{6}= & \phi\left(\frac{A_{3}^{2}}{A_{1}}\right)-\left(\frac{A_{4}^{2}}{n^{\prime} A_{2}}\right), \\
H_{7}= & \delta \gamma_{0.12}^{* 2}-\theta \rho^{* 2}, \\
H_{8}= & \left(\alpha_{3}^{2}-1\right) A_{1}^{*}+\alpha_{4}^{2} A_{2}^{*} \\
& -2\left(\alpha_{3}-1\right) A_{3}^{*}+2 \alpha_{4} A_{4}^{*}+2 \alpha_{3} \alpha_{4} A_{5}^{*}, \\
H_{9}= & H+C_{1}^{*}+M_{1}^{2} C_{2}^{*}+2 M_{1} C_{3}^{*} .
\end{aligned}
$$

$$
\begin{gathered}
N=80, \quad n=10, \quad \lambda_{400}=2.2667, \\
\lambda_{040}=3.6500, \quad \lambda_{004}=2.8664, \\
\lambda_{220}=2.3377, \quad \lambda_{202}=2.2208, \\
\lambda_{022}=3.1400 .
\end{gathered}
$$

The MSE and percent relative efficiency (PRE) of various estimators of $S_{y}^{2}$, with respect to the conventional estimator, $t_{0}$, have been computed and presented in Table 1 . Note that, for the calculation of the MSE of $t^{*}$, we take $n^{\prime}=25$ and also note that the minimum MSE of $t_{2}$ and $t_{2}^{*}$ is obtained using $\operatorname{MSE}_{\text {min }}\left(t_{2}\right)=S_{y}^{4}\left(C_{1}-C_{3}^{2} / C_{2}\right)$ and $\operatorname{MSE}_{\text {min }}\left(t_{2}^{*}\right)=S_{y}^{4}\left(D_{1}-\right.$ $\left.D_{3}^{2} / D_{2}\right)$.

Table 1 reveals that the suggested estimator $t$ has the smallest MSE and thus the highest PRE among other estimators considered in this study. The suggested estimator in TPS $t^{*}$ also provides a sufficient improvement in variance estimation compared to the existing ones $\left(t_{1}^{*}\right.$ and $\left.t_{2}^{*}\right)$. It is also observed from Table 1 that the TPS estimators are less efficient than their corresponding.

\section{Conclusion}

We have developed a new estimator for estimating the finite population variance under SRSWOR, which is found to be more efficient than the traditional estimator, Isaki [1] ratio estimators (using single and double auxiliary variables), Gupta et al. [14] estimator, and Singh et al. [8] estimator when certain conditions, as outlined in Section 4, are satisfied. This theoretical inference is also supported by the result of an application with original data. In future, we hope to extend the estimators suggested here for the development of a new estimator in the stratified random sampling. 


\section{Conflict of Interests}

The authors declare that there is no conflict of interests regarding the publication of this paper.

\section{References}

[1] C. T. Isaki, "Variance estimation using auxiliary information," Journal of the American Statistical Association, vol. 78, no. 381, pp. 117-123, 1983.

[2] B. Prasad and H. P. Singh, "Some improved ratio-type estimators of finite population variance in sample surveys," Communications in Statistics, vol. 19, no. 3, pp. 1127-1139, 1990.

[3] B. Prasad and H. P. Singh, "Unbiased estimators of finite population variance using auxiliary information in sample surveys," Communications in Statistics, vol. 21, no. 5, pp. 1367-1376, 1992.

[4] R. S. Biradar and H. P. Singh, "An alternative to ratio estimator of population Variance," Assam Statistical Review, vol. 8, no. 2, pp. 18-33, 1994.

[5] M. Rueda Garcia and A. Arcos Cebrian, "Repeated substitution method: the ratio estimator for the population variance," Metrika, vol. 43, no. 2, pp. 101-105, 1996.

[6] A. Arcos, M. Rueda, M. D. Martínez, S. González, and Y. Román, "Incorporating the auxiliary information available in variance estimation," Applied Mathematics and Computation, vol. 160, no. 2, pp. 387-399, 2005.

[7] C. Kadilar and H. Cingi, "Improvement in estimating the population mean in simple random sampling," Applied Mathematics Letters, vol. 19, no. 1, pp. 75-79, 2006.

[8] H. P. Singh, S. Singh, and J. M. Kim, "Efficient use of auxiliary variables in estimating finite population variance in two-phase sampling," Communications of the Korean Statistical Society, vol. 17, no. 2, pp. 165-181, 2010.

[9] I. Olkin, "Multivariate ratio estimation for finite populations," Biometrika, vol. 45, pp. 154-165, 1958.

[10] S. R. Srivastava, S. R. Srivastava, and B. B. Khare, "Chain ratio type estimator for ratio of two population means using auxiliary characters," Communications in Statistics, vol. 18, no. 10, pp. 3917-3926, 1989.

[11] L. N. Upadhyaya, K. S. Kushwaha, and H. P. Singh, "A modified chain ratio-type estimator in two-phase sampling using multi auxiliary information," Metron, vol. 48, no. 1-4, pp. 381-393, 1990.

[12] V. K. Singh, H. P. Singh, H. P. Singh, and D. Shukla, "A general class of chain estimators for ratio and product of two means of a finite population," Communications in Statistics, vol. 23, no. 5, pp. 1341-1355, 1994.

[13] L. Chand, Some ratio-type estimators based on two or more auxiliary variables [Ph.D. thesis], Iowa State University, Ames, Iowa, USA, 1975.

[14] R. K. Gupta, S. Singh, and N. S. Mangat, "Some chain ratio type estimators for estimating finite population variance," Aligarh Journal of Statistics, vol. 12-13, pp. 65-69, 1992-1993.

[15] W. A. Abu-Dayyeh, M. S. Ahmed, R. A. Ahmed, and H. A. Muttlak, "Some estimators of a finite population mean using auxiliary information," Applied Mathematics and Computation, vol. 139, no. 2-3, pp. 287-298, 2003.

[16] M. N. Murthy, Sampling Theory and Methods, Statistical Publishing Society, Calcutta, India, 1967. 


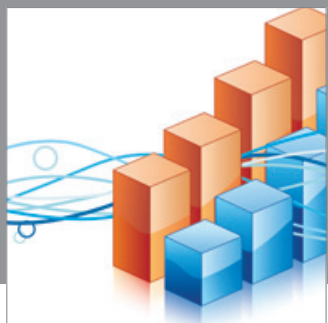

Advances in

Operations Research

mansans

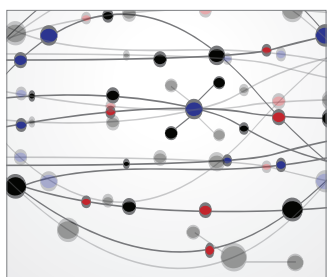

The Scientific World Journal
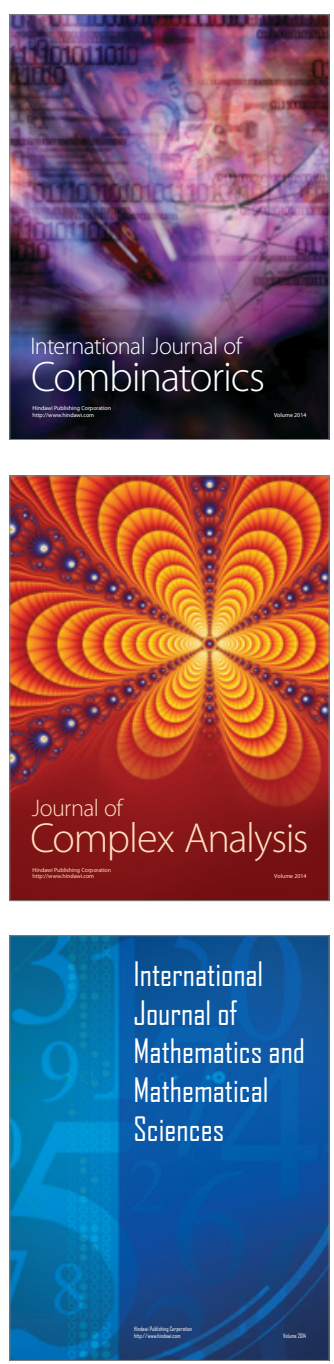
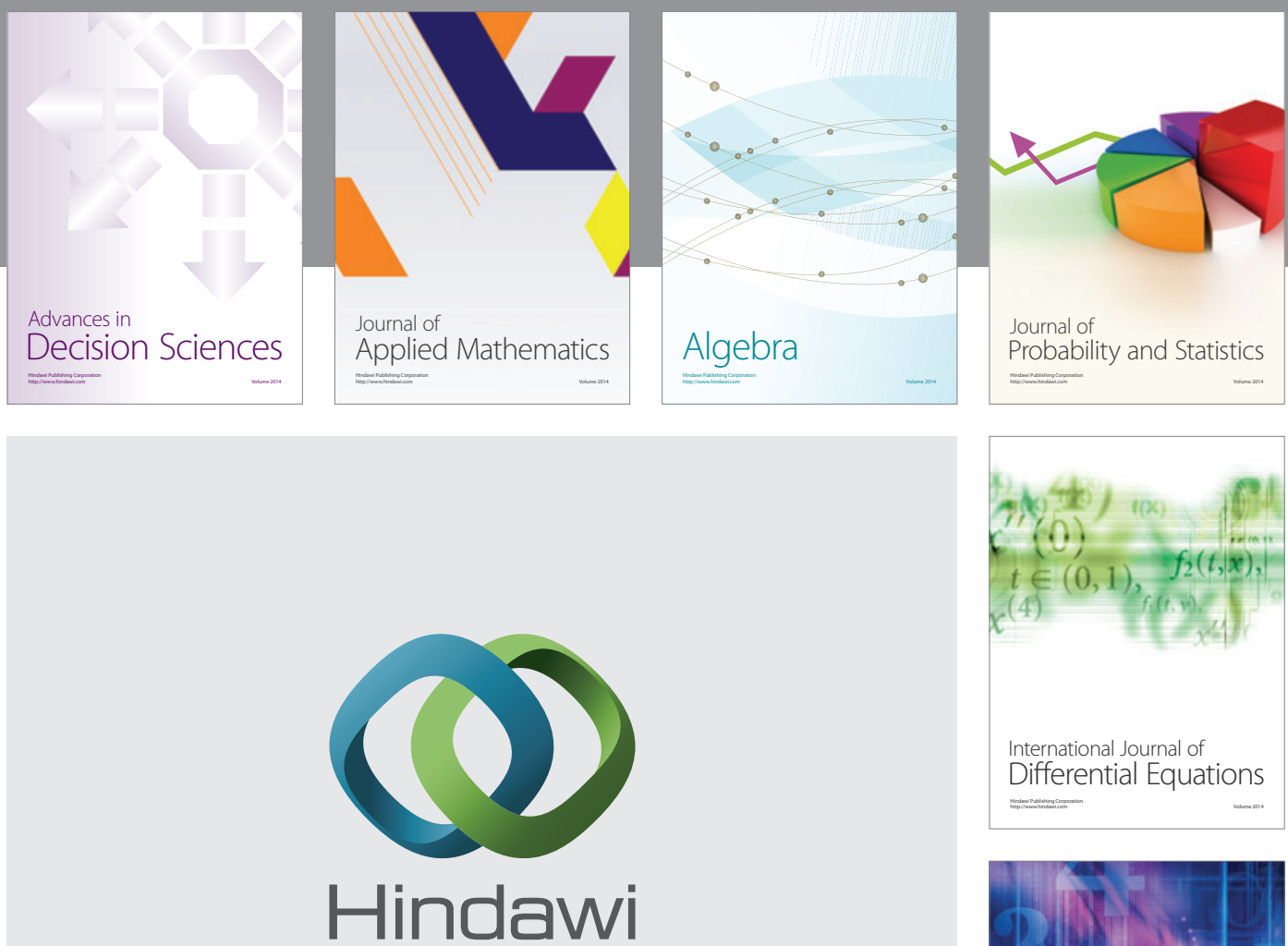

Submit your manuscripts at http://www.hindawi.com
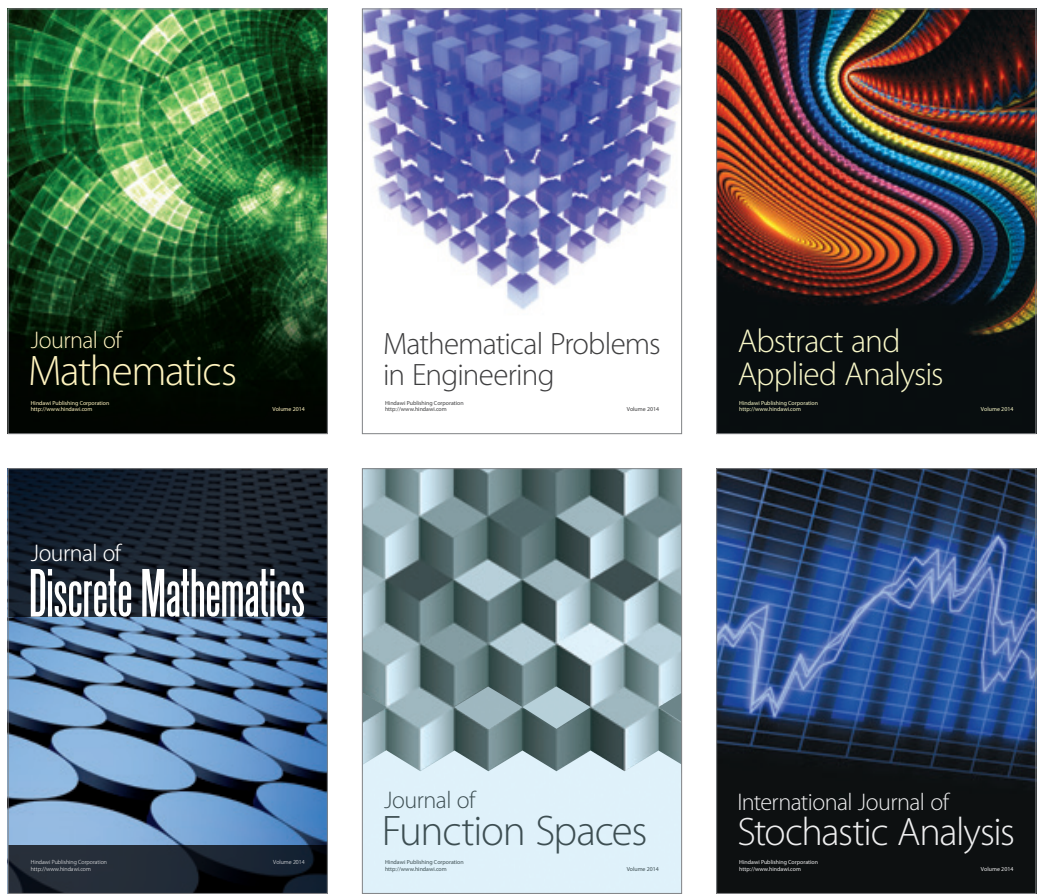

Journal of

Function Spaces

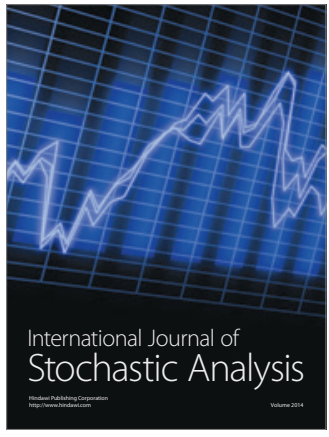

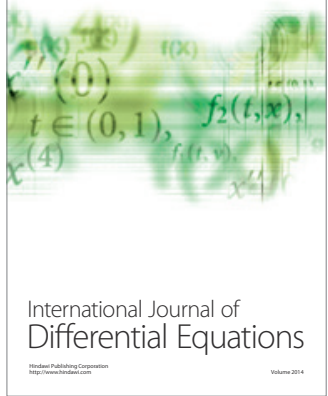
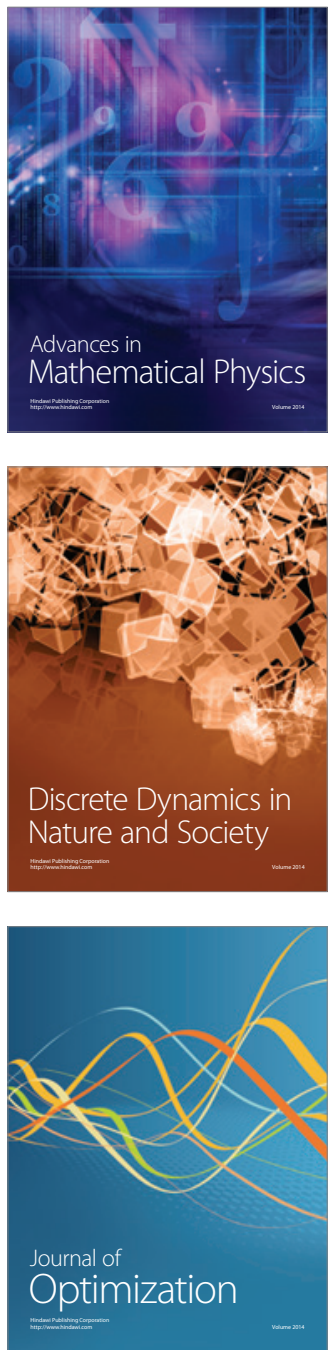